\title{
Long-term prognosis of short QT interval in Korean patients: a multicenter retrospective cohort study
}

\author{
Dae-Young Kim ${ }^{1+}$, Jae-Sun Uhm² ${ }^{2 \dagger}$, Min Kim ${ }^{1}$, In-Soo Kim', Moo-Nyun Jin ${ }^{1,3}$, Hee Tae Yu', Tae-Hoon Kim', \\ Jong-Youn Kim ${ }^{4}$, Boyoung Joung ${ }^{1}$, Hui-Nam Pak ${ }^{1}$ and Moon-Hyoung Lee ${ }^{1^{*}}$ (D)
}

\begin{abstract}
Background: Short QT syndrome is a rare, inherited channelopathy associated with sudden cardiac arrest (SCA) but the characteristics and prognosis of short QT interval (SQTI) in Korean patients remain unclear. This study aimed to determine the clinical characteristics and outcomes of SQTI in a Korean population.

Methods: Consecutive patients with SQTI from January 1999 to March 2019 in three university hospitals in South Korea were recruited. SQTI was defined as a Bazett's formula-corrected QT interval (QTc) $\leq 340$ ms in serial electrocardiograms. Age- and sex-matched patients with a normal QTc and without overt cardiovascular disease were included in a 1:4 ratio. Clinical and ECG features and outcomes were compared between patients with and without SQTI.

Results: 34 patients with SQTI [age, 23.5 (21-30.5) years; 31 male] were followed up for 4.8 (2.0-7.8) years. Early repolarization, tall T wave, and U wave were significantly more frequent in patients with SQTI than in those without SQTI. QT dispersion [44.0 (28.0-73.0) vs. $20.0(12.0-35.0) \mathrm{ms}, P<0.001]$ was significantly wider and heart rate [52.0 (47.0-58.0) vs. $70.0(62.3-84.0) / \mathrm{min}, P<0.001]$ was significantly slower in patients with SQTI than in those without. Atrial fibrillation (AF, $11.8 \%$ vs. $2.2 \%, P=0.030)$ and ventricular arrhythmia (VA)/SCA ( $8.7 \%$ vs. $0 \%, P=0.007)$ were significantly more frequent in patients with SQTI than in those without. SQTI was significantly associated with AF [odds ratio, 5.911; $95 \%$ confidence interval, 1.257-27.808; $P=0.025]$ and VA/SCA.
\end{abstract}

Conclusions: In this subset of Korean population, SQTI was associated with AF and VA/SCA.

Keywords: Atrial fibrillation, QT interval, Short QT syndrome, Sudden cardiac arrest, Ventricular arrhythmia

\section{Background}

Short QT syndrome (SQTS) is a rare, life-threatening, inherited channelopathy associated with ventricular arrhythmia (VA) and sudden cardiac arrest (SCA) [1]. The first reported clinical case was of familial short QT interval $<300 \mathrm{~ms}$ accompanied by arrhythmia [2]. After

\footnotetext{
*Correspondence: mhlee@yuhs.ac

${ }^{\dagger}$ Dae-Young Kim and Jae-Sun Uhm contributed equally to this work

${ }^{1}$ Division of Cardiology, Department of Internal Medicine, Severance

Cardiovascular Hospital, Yonsei University College of Medicine, 50-1

Yonsei-ro Seodaemun-gu, Seoul 03722, Republic of Korea

Full list of author information is available at the end of the article
}

a while, familial sudden cardiac death (SCD) accompanied with short QT interval and a history of syncope or palpitations was reported [3]. Later, SQTS was found to increase the risk of SCD, especially in young patients who were not previously diagnosed with any disease. The clinical and electrocardiogram (ECG) features, as well as clinical outcomes, remain unclear in Korean patients with SQTS. This is due to the rarity of the condition and the relatively short time since its discovery in Western countries. The aim of our study was to determine the clinical characteristics and outcomes of patients with short QT interval (SQTI) in a Korean population. 


\section{Methods}

\section{Study population}

This was a multicenter retrospective cohort study. The study design was approved by the Institutional Review Board of Yonsei University Health System (IRB approval number: 4-2019-0644), and the study was conducted in accordance with the Declaration of Helsinki. The institutional review board waived both the need for the acquisition of informed consent from patients to be included in the analysis and the need for review by a critical event committee owing to the study's retrospective nature and the absence of patient identification data.

We included consecutive patients with SQTI from January 1999 to March 2019 in three university hospitals in South Korea. SQTI was defined as a Bazett's formula-corrected QT interval (QTc) $\leq 340 \mathrm{~ms}$ in serial ECGs regardless of symptoms or history of familial SCD. For estimating the QT interval, we used GE Healthcare's 12SL software (Milwaukee, WI, USA). A representative beat was generated from the selected segment of each lead, and then a composite beat was formed by the representative beats of all independent leads. The global QT interval was considered as the longest QT interval in the multiple leads. Patients of all ages who met the SQTI definition were included in the SQTI group, even in cases without related symptoms or a history of familial SCD (Fig. 1). The Exclusion criteria were as follows: temporary SQTI due to electrolyte imbalance (e.g. hypercalcemia, hyperkalemia), acidosis, hyperthermia, use of digitalis and cholinergic drugs which were related to clinical impairments, especially during hospitalization; structural heart disease with ischemic or nonischemic cardiomyopathy; and significant (high-degree and third-degree) atrioventricular block (AVB). Age- and sex-matched subjects in the normal QT group were recruited in a 1:4 ratio and had at least three ECGs with normal QTc which were performed from January 1999 to March 2019 in the outpatient clinic. Patients with overt cardiovascular disease, defined as cardiomyopathy, coronary artery disease, and significant bradyarrhythmia, were excluded. Clinical outcomes included arrhythmia outcomes and all-cause death. Arrhythmia outcomes included atrial fibrillation (AF), VA/SCA, and Mobitz type $2 \mathrm{~s}-$, high-, and thirddegree AVB.

\section{Data analysis}

The following clinical variables were collected from all recruited subjects: age, sex, height, weight, systolic and diastolic blood pressure, clinical history of loss of consciousness, palpitations, chest pain, SCA, the list of medications, familial history of SCA, accompanying arrhythmias (AF, VA, premature ventricular complex, supraventricular tachycardia, first-degree AVB, second-degree AVB, high-degree AVB, third-degree $\mathrm{AVB}$ ), and ECG parameters (PR interval, QT interval, QT dispersion, heart rate, JT interval, biphasic negativepositive $\mathrm{T}$ wave, early repolarization, and tall $\mathrm{T}$ waves). Tall $\mathrm{T}$ waves were defined as $>10 \mathrm{~mm}(1 \mathrm{mV})$ in any of the precordial leads.[4] QT dispersion was calculated as the difference between the maximum and minimum QT interval in the 12-lead ECG. JT interval was measured from the J point to the $\mathrm{T}$ wave peak. Early repolarization was defined as an elevation of the QRS-ST junction (J point) in leads other than V1-V3.[5]

We compared the baseline characteristics including symptoms, family history, ECG findings, and clinical outcomes between the two groups.

\section{Statistical analysis}

The results are presented as median (interquartile range) for continuous data and as frequency (percentage) for categorical data. To compare the clinical parameters between groups, we used the Mann Whitney U-test for continuous data and Fisher's exact test for categorical data since all data sets were non-normally distributed. Univariate and multivariate logistic regression analyses were performed to determine the odds ratio of arrhythmia in patients with SQTI. A p-value $\leq 0.05$ was considered statistically significant. Data were analyzed using Statistical Package for the Social Sciences version 25.0 (IBM Corporation, Armonk, NY).

\section{Results}

A total of 4,694,382 ECGs were performed in 2,083,360 patients (both inpatients and outpatients) during the study period; 34 patients with SQTI were found, corresponding to a prevalence of $0.001 \%$. These 34 patients [age, 23.5 (21.0-30.5) years; 31 male] with SQTI were followed up for $4.9(2.0-7.8)$ years. Table 1 shows the baseline characteristics, medications, symptoms, and ECG characteristics of patients in the SQTI and normal QT groups. In the SQTI group, males younger than 40 years were dominant $(91.2 \%)$. The medications in the SQTI and normal QT groups were not significantly different except for digoxin (5.9\% vs. $0.7 \%, P=0.041)$; drugs that were known to shorten QT interval were not administered to patients in the SQTI and normal QT groups. Among the 34 patients with SQTI, 19 patients $(55.9 \%)$ visited the hospitals for the evaluation of symptoms such as chest pain, chest discomfort, dyspnea, palpitations, and dizziness. The others had various causes of visiting the hospitals [hospitalization for other causes $(n=8,23.5 \%)$, routine health checkup $(\mathrm{n}=4,11.8 \%)$, preoperative evaluation for noncardiac surgery $(n=3,8.8 \%)$ in order of frequency]. Symptoms included palpitations $(n=4,11.8 \%)$, loss 


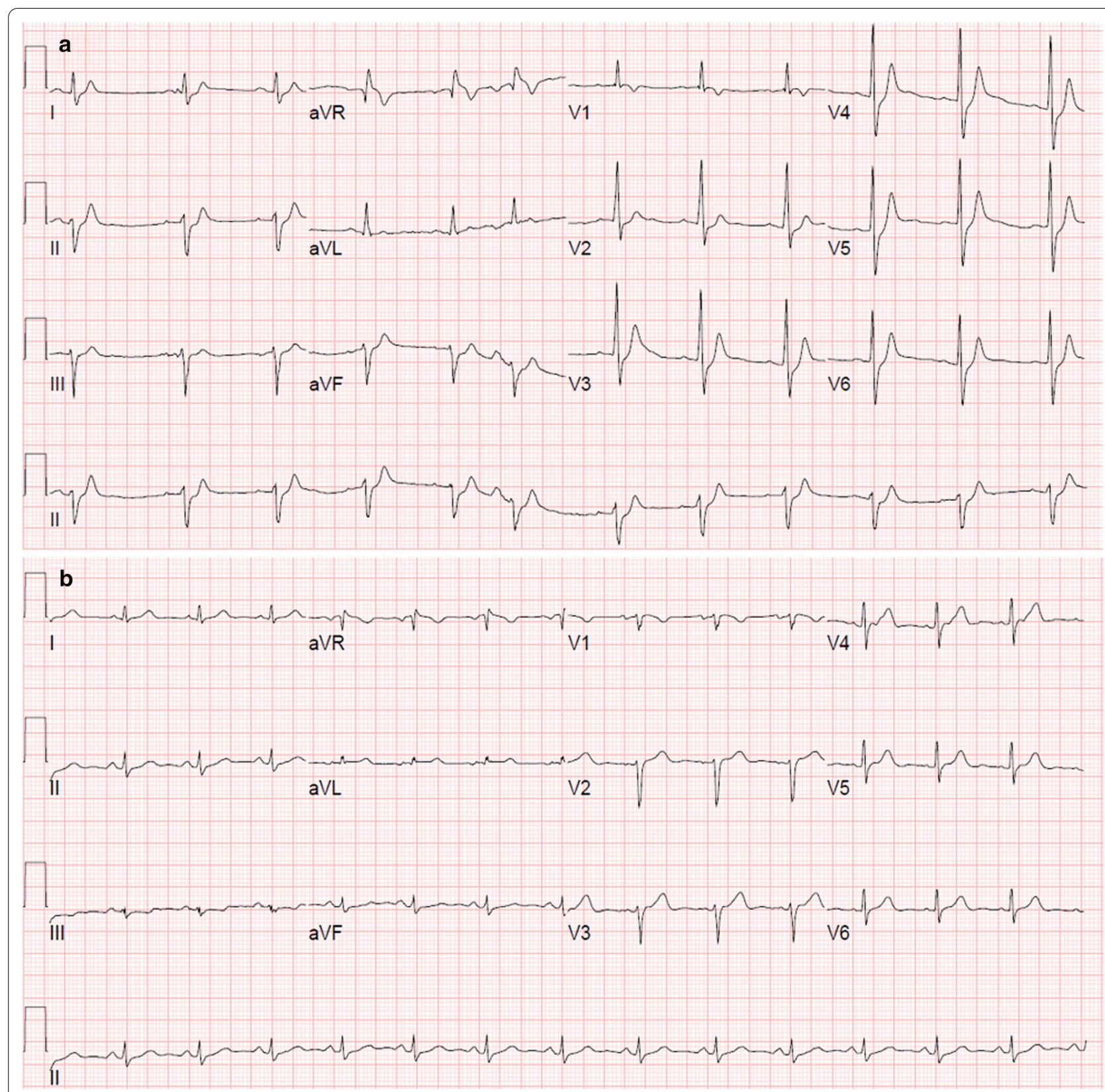

Fig. 1 Representative twelve-lead ECG. A) A patient with short QT syndrome who experienced sudden cardiac arrest. QT interval, 306 ms; corrected QT interval, 330 ms; QRS duration, 88 ms; and and heart rate, 70/min. B) A patient with normal QTc without any clinical history who visited the outpatient clinic due to upper respiratory infection. QT interval, 366 ms; corrected QT interval, 432 ms; QRS duration, 94 ms; and heart rate, 84/min

of consciousness $(\mathrm{n}=3,8.8 \%)$, and chest pain $(\mathrm{n}=3$, $8.8 \%)$. Palpitations $(11.8 \%$ vs. $2.2 \%, P=0.030)$ were significantly more frequent in the SQTI group than in the normal QT group. Regarding ECG findings, QT dispersion was significantly wider and heart rate was significantly slower in the SQTI group than in the normal QT group. Early repolarization $(\mathrm{n}=25,73.5 \%)$, tall $\mathrm{T}$ waves $(n=20,58.8 \%)$, and $U$ waves $(n=4,11.8 \%)$ were significantly more frequent in the SQTI group than in the normal QT group. There were no significant differences in the frequencies of premature ventricular contraction and supraventricular tachycardia between groups. Among patients with SQTI, twelve patients underwent transthoracic echocardiography during the follow-up period, which revealed no structural cardiac abnormalities. 
Table 1 Baseline characteristics of the SQTI and normal QT groups

\begin{tabular}{|c|c|c|c|}
\hline & SQTI group $(n=34)$ & Normal QT group $(n=136)$ & $P$ \\
\hline \multicolumn{4}{|l|}{ Demographics } \\
\hline Age (year) & $23.5(21.0-30.5)$ & $23.5(21.0-31.0)$ & $>0.999$ \\
\hline Male & $31(91.2)$ & $124(91.2)$ & $>0.999$ \\
\hline Family history of SCA & $1(2.9)$ & $0(0.0)$ & 0.200 \\
\hline Height (cm) & $173.0(170.0-176.8)$ & $173.0(168.0-177.0)$ & 0.698 \\
\hline Weight (kg) & $66.2(61.1-72.0)$ & $69.5(60.0-78.8)$ & 0.155 \\
\hline Systolic BP (mmHg) & $124.0(113.3-129.0)$ & $122.0(113.3-134.0)$ & 0.537 \\
\hline Diastolic BP (mmHg) & $71.0(66.0-78.0)$ & $76.0(67.3-83.0)$ & 0.056 \\
\hline ICD implantation & $1(2.9)$ & $0(0.0)$ & 0.200 \\
\hline \multicolumn{4}{|l|}{ Medications } \\
\hline Digoxin & $2(5.9)$ & $1(0.7)$ & 0.041 \\
\hline$\beta$-blocker & $3(8.8)$ & $7(5.1)$ & 0.415 \\
\hline Calcium channel blocker & $5(14.7)$ & $7(5.1)$ & 0.052 \\
\hline RASi & $3(8.8)$ & $6(4.4)$ & 0.304 \\
\hline Antiarrhythmic drug & $2(5.9)$ & $2(1.5)$ & 0.129 \\
\hline Amiodarone & $1(2.9)$ & $1(0.7)$ & \\
\hline Flecainide & $1(2.9)$ & $1(0.7)$ & \\
\hline Diuretics & $3(8.8)$ & $7(5.1)$ & 0.415 \\
\hline Lipid lowering agent & $1(2.9)$ & $2(1.5)$ & 0.560 \\
\hline Antiplatelet & $1(2.9)$ & $3(2.2)$ & 0.800 \\
\hline Anticoagulant & $1(2.9)$ & $5(3.7)$ & 0.835 \\
\hline \multicolumn{4}{|l|}{ Symptoms } \\
\hline Loss of consciousness & $3(8.8)$ & $4(2.9)$ & 0.144 \\
\hline Palpitations & $4(11.8)$ & $3(2.2)$ & 0.030 \\
\hline Chest pain & $3(8.8)$ & $8(5.9)$ & 0.461 \\
\hline \multicolumn{4}{|l|}{ ECG characteristics } \\
\hline Heart rate (/min) & $52.0(47.0-58.0)$ & $70.0(62.3-84.0)$ & $<0.001$ \\
\hline PR interval (ms) & $160.0(146.0-175.5)$ & $152.0(137.5-168.0)$ & 0.061 \\
\hline QT interval (ms) & $361.0(335.0-375.5)$ & $380.0(358.0-400.0)$ & 0.001 \\
\hline QTc (ms) & $334.0(330.3-337.0)$ & $416.0(396.3-436.8)$ & $<0.001$ \\
\hline QT dispersion (ms) & $44.0(28.0-73.0)$ & $20.0(12.0-35.0)$ & $<0.001$ \\
\hline JT interval (ms) & $262.0(245.0-279.0)$ & $200.0(169.0-224.0)$ & $<0.001$ \\
\hline U wave & $4(11.8)$ & $0(0)$ & $--^{\mathrm{a}}$ \\
\hline Biphasic T wave & $1(2.9)$ & $0(0)$ & $-^{\mathrm{a}}$ \\
\hline Early repolarization & $25(73.5)$ & $14(10.3)$ & $<0.001$ \\
\hline Tall T waves & $20(58.8)$ & $10(7.4)$ & $<0.001$ \\
\hline PVC & $2(5.8)$ & $2(1.5)$ & 0.179 \\
\hline SVT & $2(5.8)$ & $2(1.5)$ & 0.179 \\
\hline Follow-up period (year) & $4.9(2.0-7.8)$ & $3.8(3.8-4.2)$ & 0.318 \\
\hline
\end{tabular}

The results are presented as median (interquartile range) for continuous data and as frequency (percentage) for categorical data

$B P$ blood pressure, ICD implantable cardioverter-defibrillator, RASi renin angiotensin system inhibitor, PVC premature ventricular contraction, SQTI short QT interval, SVT supraventricular tachycardia

a Statistical comparison could not be performed because the number of patients was small

Table 2 shows the arrhythmia outcomes. AF (11.8\% vs. $2.2 \%, P=0.030$ ) was significantly more frequent in the SQTI group than in the normal QT group. Three patients experienced VA/SCA in the SQTI group, whereas no patients experienced VA/SCA in the normal QT group
(8.7\% vs. $0 \%, P=0.007)$. One patient had ventricular fibrillation and underwent cardioverter-defibrillator implantation (Fig. 1a). Another patient had ventricular tachycardia but he refused cardioverter-defibrillator implantation and was lost to follow up. The other patient 
Table 2 Clinical outcomes in the SQTI and normal QT groups

\begin{tabular}{llll}
\hline & $\begin{array}{l}\text { SQTI group } \\
(\mathbf{n = 3 4 )}\end{array}$ & $\begin{array}{l}\text { Normal QT group } \\
(\mathbf{n = 1 3 6 )}\end{array}$ & $\boldsymbol{P}$ \\
\hline Arrhythmia outcomes & & & \\
AF & $4(11.8)$ & $3(2.2)$ & 0.030 \\
VA/SCA & $3(8.7)$ & $0(0)$ & 0.007 \\
Significant AVB & $3(8.7)$ & $8(5.9)$ & 0.461 \\
Non-cardiac death & $0(0.0)$ & $7(5.1)$ & 0.347 \\
\hline
\end{tabular}

The results are presented as median (interquartile range) for continuous data and as frequency (percentage) for categorical data

$A F$ atrial fibrillation, AVB atrioventricular block, SCA sudden cardiac arrest, SQTI short QT interval, VA ventricular arrhythmia

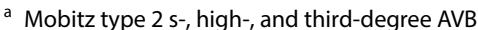

Table 3 Multivariate logistic regression analysis of factors for the occurrence of AF

\begin{tabular}{lll}
\hline & Odds ratio $(\mathbf{9 5 \%} \mathrm{Cl})$ & $\boldsymbol{P}$ \\
\hline SQTI & $7.834(1.387-44.258)$ & 0.020 \\
Age & $1.057(1.016-1.099)$ & 0.006 \\
Males & $0.286(0.040-2.029)$ & 0.211 \\
\hline
\end{tabular}

AF atrial fibrillation, SQTI short QT interval

had SCD. There were no significant differences in the occurrence of significant AVB between the groups. Multivariate logistic regression analysis adjusted for age and sex showed that SQTI was independently associated with AF [odds ratio, 7.834; 95\% confidence interval, 1.38744.258; $P=0.020$ ] and VA/SCA (a statistical comparison could not be performed because the incidence of VA/ SCA in the normal QT group was zero) (Table 3).

\section{Discussion}

\section{Main findings}

The main findings of the present study are as follows: (1) the prevalence of SQTI was 0.001\%; (2) QT dispersion was significantly wider and heart rate was significantly slower in patients with SQTI than in those with a normal QT interval. Early repolarization, tall $\mathrm{T}$ waves, and $\mathrm{U}$ waves were more frequent in patients with SQTI than in patients with normal QT interval; and (3) SQTI was significantly associated with AF and VA/SCA.

\section{SQTS diagnostic criteria}

Multiple studies have suggested diverse diagnostic criteria for SQTS; definite diagnostic criteria for SQTS have not been properly established since the condition was first identified. Initially, a constant QT interval $\leq 300 \mathrm{~ms}$ was regarded as a short QT interval.[3] After major population studies, the suggested diagnostic criteria for SQTS were a QTc $\leq 340 \mathrm{~ms}$ or a QTc $\leq 370 \mathrm{~ms}$ in the presence of a pathologic mutation; familial history of SQTS or SCA at $<40$ years of age; or a history of VA in a structurally normal heart, AF in early life, or loss of consciousness that might be strongly related to a cardiac arrhythmia [6].

\section{Sex differences in SQTI}

The majority of patients with SQTI were male and between 20 and 30 years of age. It is known that the QT interval is shorter in men than in women [7], which may be attributable to the effect of testosterone on the QTc $[8$, 9].

\section{Symptoms}

Over $35 \%$ of patients with SQTI had symptoms at presentation; these included, in decreasing frequency, palpitation $(\mathrm{n}=4,11.8 \%)$, loss of consciousness $(\mathrm{n}=3,8.8 \%)$, and chest pain $(n=3,8.8 \%)$. A previous study showed that the most frequent symptoms were palpitations and syncope, which is in accordance with the present study [10]. The cause of loss of consciousness may be self-terminating VA in patients with SQTS [11].

\section{ECG characteristics}

A previous study demonstrated that early repolarization was associated with arrhythmias in patients with SQTS and could be used for identifying the risk of SCA in SQTS [12]. Tall T wave is one of the characteristic ECG findings in SQTS [12]. QT dispersion was significantly wider in the SQTI group than in the normal QT group in the present study. It is known that increases in repolarization dispersion are the possible substrate for reentry, which may result in VA and SCA [11]. A previous study also found that patients with SQTS had significantly wider QT dispersion [13].

\section{Arrhythmia outcomes}

AF was the most frequent arrhythmia in the SQTI group in the present study. A prior study reported that $18.0 \%$ of patients with SQTS had AF [14]. Given the close association between SQTS and AF, the latter is included in the SQTS diagnostic criteria. This relationship was found to be attributable to a common KCNQ1 missense mutation (V141M), which caused AF and shortened the QT interval by altering the gating of $\mathrm{I}_{\mathrm{Ks}}$ channels [15]. Approximately $5.8 \%$ of patients experienced VA at the initial SQTI diagnosis.

\section{Limitations}

The present study has a number of limitations. First, a small number of patients were included in the present study because SQTI is a rare condition. Large-scale prospective studies of patients with SQTI are needed. 
Second, genetic studies were not performed in patients with SQTI. Third, subjects with normal QT interval were recruited not from the general population but from among hospital visitors; this was decided since ECG is not routinely performed in the general population. However, subjects with normal ECG findings and without any cardiovascular disease were included to avoid selection bias. Fourth, conditions such as a history of physical activity and emotional status, which can affect QT interval, were not assessed because of the retrospective design.

\section{Conclusions}

In this cohort of Korean population, SQTI was associated with AF and VA/SCA. It is important to note that SQTI and AF have close relationship with common KCNQ1 mutation (V141M) and when SQTI is detected by taking an ECG, it is necessary to actively monitor for syncope, $\mathrm{AF}$, and VA.

\begin{abstract}
Abbreviations
SQTS: Short QT syndrome; VA: Ventricular arrhythmia; SCA: Sudden cardiac arrest; SCD: Sudden cardiac death; ECG: Electrocardiogram; SQTI: Short QT interval; QTc: Corrected QT interval; AVB: Atrioventricular block; AF: Atrial fibrillation.
\end{abstract}

\section{Acknowledgements}

The authors thank Ju-young Park, M.S. and Soyoung Jeon, M.S. in the Biostatistics Collaboration Unit, Yonsei University College of Medicine, for their assistance with the statistical analyses and Kina Hu, M.B.A. of GE Healthcare for her assistance with data collection from MUSE ${ }^{\mathrm{TM}}$ Cardiology Information System. We have previously published the abstract of this article in the Heart Rhythm journal on May, 2020 [16].

\section{Authors' contributions}

DYK and JSU: conception and design of the study, acquisition of data, analysis and interpretation of the data, and manuscript drafting. MK, ISK, and MNJ: acquisition of data, analysis and interpretation of the data, and technical help. HTY, THK, JYK, BYJ and HNP: technical help, writing, and editing assistance. $\mathrm{MHL}$ : conception of the study, technical help, writing, and editing assistance. All authors have read and approved the final manuscript.

\section{Funding}

Not applicable.

\section{Availability of data and materials}

The datasets used and analyzed during the current study are available from the corresponding author on reasonable request.

\section{Ethics approval and consent to participate}

The study design was approved by the Institutional Review Board of Yonsei University Health System (IRB approval number: 4-2019-0644), and the study was conducted in accordance with the Declaration of Helsinki. The institutional review board waived both the need for the acquisition of informed consent from patients to be included in the analysis and the need for review by a critical event committee owing to the study's retrospective nature and the absence of patient identification data. We have got permissions to access the raw data in which personal information is anonymized, from the directors of Yonsei University's three Severance hospitals.

\section{Author details}

${ }^{1}$ Division of Cardiology, Department of Internal Medicine, Severance Cardiovascular Hospital, Yonsei University College of Medicine, 50-1 Yonsei-ro Seodaemun-gu, Seoul 03722, Republic of Korea. ${ }^{2}$ Division of Cardiology, Department of Internal Medicine, Yongin Severance Hospital, Yonsei University College of Medicine, Yongin-si, Gyunggi-do, Republic of Korea. ${ }^{3}$ Division of Cardiology, Department of Internal Medicine, Sanggye Paik Hospital, Inje University College of Medicine, Seoul, Republic of Korea. ${ }^{4}$ Division of Cardiology, Department of Internal Medicine, Gangnam Severance Hospital, Yonsei University College of Medicine, Seoul, Republic of Korea.

Received: 7 September 2020 Accepted: 14 December 2020

Published online: 06 January 2021

\section{References}

1. Giustetto C, Schimpf R, Mazzanti A, Scrocco C, Maury P, Anttonen O, et al. Long-term follow-up of patients with short QT syndrome. J Am Coll Cardiol. 2011;58:587-95. https://doi.org/10.1016/j.jacc.2011.03.038.

2. Gussak I, Brugada P, Brugada J, Wright RS, Kopecky SL, Chaitman BR, et al. Idiopathic short QT interval: a new clinical syndrome? Cardiology. 2000;94:99-102.

3. Gaita F, Giustetto C, Bianchi F, Wolpert C, Schimpf R, Riccardi R, et al. Short QT syndrome: a familial cause of sudden death. Circulation. 2003;108:965-70.

4. Scognamiglio R, Fasoli G, Bruni A, Dalla-Volta S. Observations on the capability of the electrocardiogram to detect left ventricular function in chronic severe aortic regurgitation. Eur Heart J. 1988;9:54-60.

5. Tikkanen JT, Anttonen O, Junttila MJ, Aro AL, Kerola T, Rissanen HA, et al. Long-term outcome associated with early repolarization on electrocardiography. N Engl J Med. 2009;361:2529-37.

6. Bjerregaard P. Diagnosis and management of short QT syndrome. Hear Rhythm. 2018;15:1261-7. https://doi.org/10.1016/j.hrthm.2018.02.034.

7. Bidoggia H, Maciel JP, Capalozza N, Mosca S, Blaksley EJ, Valverde E, et al. Sex-dependent electrocardiographic pattern of cardiac repolarization. Am Heart J. 2000;140(3):430-6.

8. JØrgensen IN, SkakkebÆk A, Andersen NH, Pedersen LNø, Hougaard DM, Bojesen A, et al. Short QTc interval in males with klinefelter syndromeInfluence of CAG repeat length, body composition, and testosterone replacement therapy. PACE Pacing Clin Electrophysiol. 2015;38:472-82.

9. Sedlak T, Shufelt C, Iribarren C, Merz CNB. Sex hormones and the QT interval: a review. J Women's Heal. 2012;21:933-41.

10. El-Battrawy I, Besler J, Liebe V, Schimpf R, Tülümen E, Rudic B, et al. Longterm follow-up of patients with short QT syndrome: clinical profile and outcome. J Am Heart Assoc. 2018:7:1-9.

11. Bjerregaard P, Gussak I. Short QT Syndrome 2.pdf. 2005; 436-40.

12. Watanabe H, Makiyama T, Koyama T, Kannankeril PJ, Seto S, Okamura K, et al. High prevalence of early repolarization in short QT syndrome. Heart Rhythm. 2010;7:647-52.

13. Frea S, Giustetto C, Capriolo M, Scrocco C, Fornengo C, Benedetto S, et al. New echocardiographic insights in short QT syndrome: more than a channelopathy? Heart Rhythm. 2015;12:2096-105. https://doi. org/10.1016/j.hrthm.2015.05.024.

14. Gollob MH, Redpath CJ, Roberts JD. The short QT syndrome: proposed diagnostic criteria. J Am Coll Cardiol. 2011;57:802-12.

15. Hong K, Piper DR, Diaz-Valdecantos A, Brugada J, Oliva A, Burashnikov E, et al. De novo KCNQ1 mutation responsible for atrial fibrillation and short QT syndrome in utero. Cardiovasc Res. 2005;68:433-40.

16. Uhm J, Kim M, Kim IS, Jin MN, Yu HT, Lee MH, et al. Long-term prognosis of Asian patients with short QT interval. Heart Rhythm. 2020. https:// www.heartrhythmjournal.com/article/S1547-5271(20)30301-5/pdf.

\section{Publisher's Note}

Springer Nature remains neutral with regard to jurisdictional claims in published maps and institutional affiliations.

\section{Consent for publication}

Not applicable.

\section{Competing interests}

The authors declare that they have no competing interests. 